\title{
Urban Road Change Detection using Morphological Processing
}

\author{
Ei Phyu Sin Win \\ Department of Information Technology Engineering \\ Technological University (Kyaukse) \\ Kyaukse, Upper Myanmar \\ panthakhin9001@gmail.com \\ https://doi.org/10.48161/qaj.v1n1a29
}

\begin{abstract}
The primary point of this research is to design a road extraction algorithm for processing National Aeronautics and Space Administration satellite pictures. Roadway network detection is one of the important appointments for calamity emergency response, smart shipping structures, and real-time modify roadway network. Everyone is trying to detect road; this system is useful for urban or rural developing schedule. The development of a town / village depends not only on the building and population density of the town or village, but also in the systematic development of roads. The research focused on finding ways to use morphological image processing primarily. As an application area, we use National Aeronautics and Space Administration imagery obtained from 2009-2020 in Monywa, Upper Myanmar to find out how the roads have been developed and how the city has been developed. Extraction road from planet pictures is hard matter with many realistic application programs. The primary points in the model are the advancement of the picture, the segmentation of that picture, the application of the morphological operators, and finally the detection of the roadway network. Use Google Earth Pro to get the necessary data photos and search for road improvements. After collecting images from different seasons and years, we can find precise answers by combining them with precise algorithms. In addition to significant, benefits of Google Earth Pro, this research demonstrates the ability to make good use of satellite imagery and to integrate it with outside experts to save money, save time, and provide accurate answers. It is simulated with MATLAB programming language.
\end{abstract}

Keywords-satellite, NASA, urban, developing, schedule, morphological processing.

\section{INTRODUCTION}

Roadway detection is one of the primary characters in many applications respect the improvement of present humanity existence. So, the requirement for road detection using a strong and effective technique is also superior.

In the last decade, the road network shifts at a fast rate due to urban growth. So, it is difficult to hold the accuracy and exactness of the roadway system. To implement large application programs such as town project and governance system, automatic road detection for updating metropolis map has past come to a popularity experiment topic. It can be used for mapping in cities. A city planning, and Geographic Information System (GIS) is used. Roads are one of the most important artificial parts in any nation. So, the roadway network needs to be updated exactly and precisely to hold the precision of the roadway system for traffic governance, military decision- making, emergency management, vehicle navigation, etc. Manual extraction methods from satellite and aerial images were very time consuming and accurate, as they were not available with automatize methodology. Presently, there are many ways to detect roadway automatically. Some proposed methods are explained clearly in the next section.

This is the basis for the introduction of innovative technologies and methods such as management road traffic. Satellite imagery is indispensable resource for providing geographic information, regardless of topography or human living conditions. In short, it simplifies human life by being aware of all aspects of life. Satellite imagery and remote sensing provide numerical and characterization data, simplifying field work and learning time. The roadmap is now manually updated using high resolution aerial imagery. There are many researchers who are interested in finding a way. Some have suggested the Hough Transformation to use the "snake" method to find roads and connect broken roads.

To detect road the author uses morphological operations in this journal due to detect a wide range of roads using this method.

To pass the difficult problems and to make a hard toward an unsupervised road features extraction model, various methods are mixed. Many processing steps such as that each step is a requirement for the success of the succeeding. The fast step is to improve the extraction of information by utilizing many various picture improvement methods. The next steps in the design use of an unsupervised classification technique to separate the road features from NASA picture. Then the system applies to improve morphological operator extraction. 


\section{LITERATURE SURVEY}

The researchers pointed as an improvement over the standard U-Net architecture, and they used similar networks with pre-trained encoders. Our network has a UNet-like architecture that uses pretrained ResNet-34 [1] networks as an encoder. The encoder starts with the initial block that performs convolution with a kernel of size $7 \times 7$ and stride 2 at 2015 .

The output of the model is a pixel-by pixel mask that shows the class of each pixel. They used a slightly modified version of the original U-Net model that previously proved itself very useful for segmentation problems with limited amounts of data, see [2].

The scientists searched that is to propose a novel road extraction method that can efficiently extract the road network from remote sensing imagery with local and global information. A dataset from Google Earth was used to validate the method, and experiments showed that the proposed deep convolutional neural network can extract the road network accurately and effectively. This method

also achieves a harmonic mean of precision and recall higher than other machine learning and deep learning methods in September, 2018. [3]

In this work, they leveraged that advances and propose a deep learning-based solution capable of efficiently extracting the surface area of secondary roads at a large scale. The solution is based on hybrid segmentation models trained with high-resolution remote sensing imagery divided in tiles of $256 * 256$ pixels and their correspondent segmentation masks, resulting in increases in performance metrics of $2.7-3.5 \%$ when compared to the original architectures, 17 October 2020.[4]

The road extraction process can perform one or more operations, such as image segmentation (classification method), line segments of a certain width (Huff transforms and edges detector), contours of objects based on contours, merging associated with removing droplets. Road segments (morphological work), similarity to road patterns, etc.

\section{DESIGN AND Methodology}

In this section, we will cover the structure of the system, how to capture images from NASA, and how to get rid of roads.

\section{III.(a) Picture Acquisition from NASA}

Google Earth Pro gets the users fly anywhere on Earth to see satellite pictures, maps, terrain, 3D buildings, from galaxies in outer space to the ravine of the ocean. The users can investigate abundant earth subject, save the users' journey points, and share with others.

Satellites send pictures and another valuable data with big antennas. It also receives details where they are and how they are doing as the following Figure.

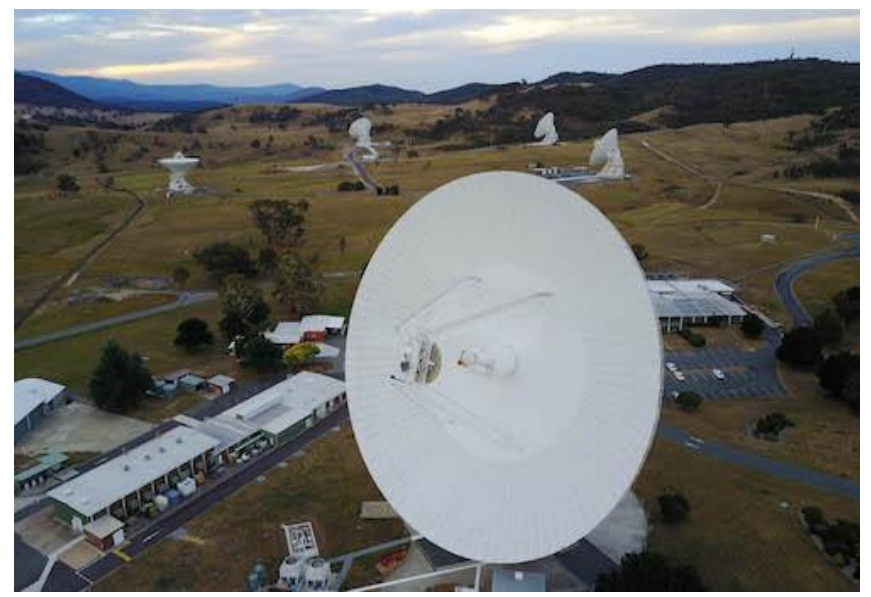

Fig. 1. NASA ' Big Antennas [5]

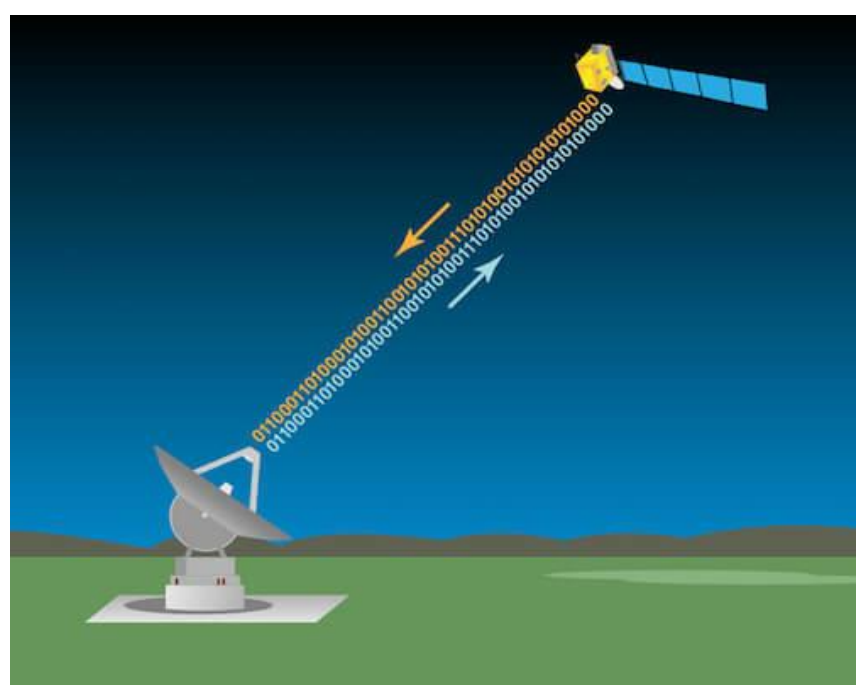

Fig. 2. Satellite sending and Antenna Receiving [6]

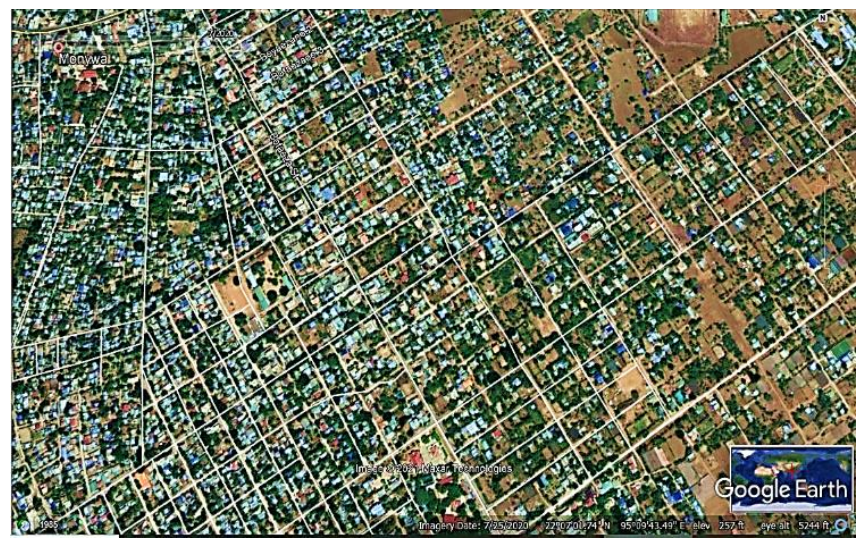

Fig. 3. Image captured on Monywa Township in Myanmar

at (7/2020) 22॰06' 57.59" N 95॰10' 10.87" E elev 258ft eye alt $5244 \mathrm{ft}$

III.(b) System Design 


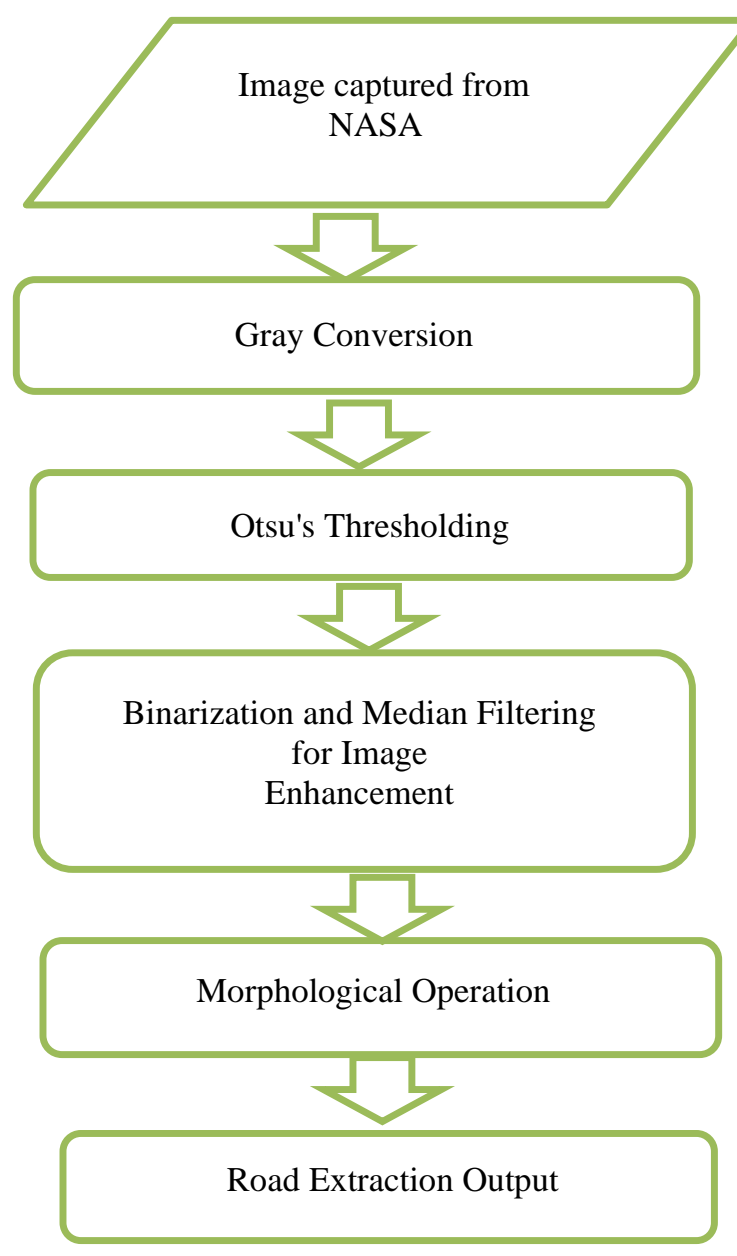

Fig. 4. System Flow Diagram

\section{III.(c)Gray Conversion}

There are several commonly used methods for converting RGB images to grayscale images, such as the averaging and weighting methods.

The average method takes the average value of Red, Green, and Blue as the grayscale value as the following theoretically equation;

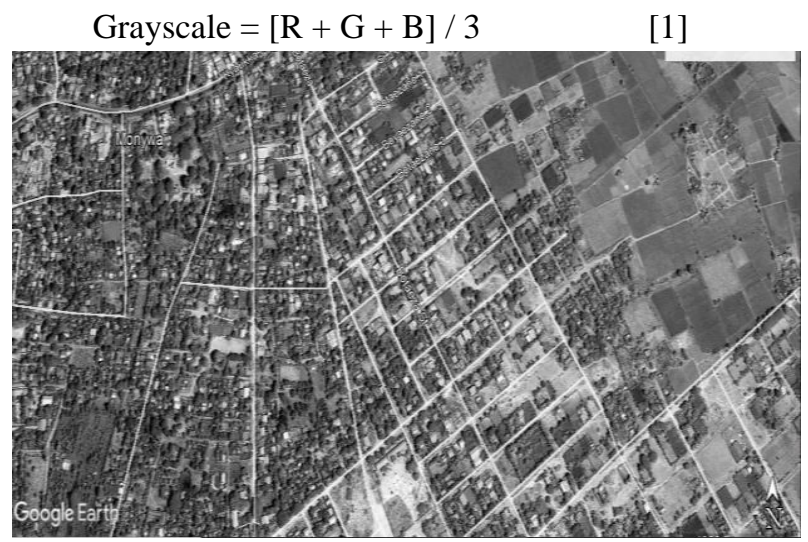

Fig.5. Gray Picture of Test Monywa

\section{III.(d) Otsu's Thresholding}

It is the simplest way to distinguish between foreground and background. It is the most powerful and convenient way to split an image. Thresholding is used to convert gray images into binary images. This has the advantage of simplifying information and reducing the complexity of the memorization process

The ordinary method to convert grayscale pictures to binary pictures is to select a single gateway value (T), $\mathrm{T}$ is set to black background (0) above, $\mathrm{T}$ is white (1).[7]

Thresholding operation is defined as the following;

$g(x, y)=\left[\begin{array}{cc}0 & \text { if } f(x, y)<T \\ 1 & \text { if } f(x, y) \geq T\end{array}\right.$

where $(\mathrm{x}, \mathrm{y}) \quad=$ a gray value

$\mathrm{T}=$ Threshold value

$\mathrm{g}(\mathrm{x}, \mathrm{y})=$ Threshold Picture

$\mathrm{f}(\mathrm{x}, \mathrm{y})=$ gray level picture pixel

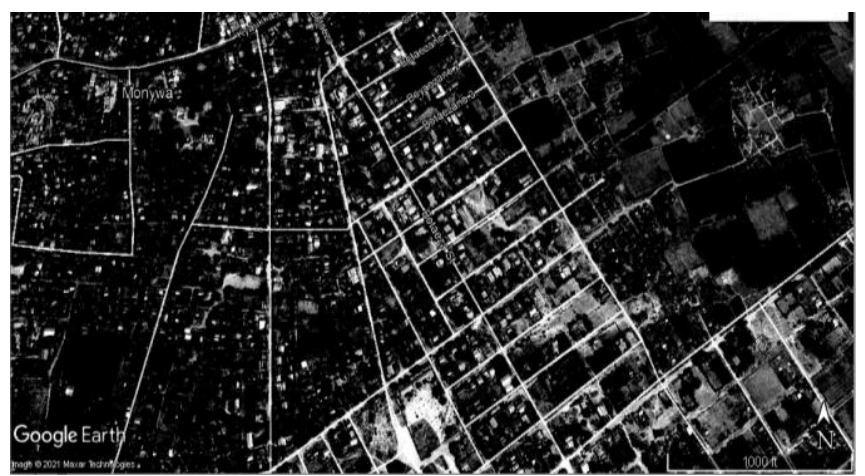

Fig. 6. Otsu's Thresholding Picture of Test Monywa

\section{III. (e) Median Filtering}

The filters are based on the order of the pixel values that make up the specified distance by providing statistical filtering equipment. Some useful spatial filters are average filters; Maximum and minimum filters; midpoint filters and trimmed alpha filters. [8] Median filter is

$$
\widehat{f}(\mathrm{x}, \mathrm{y})=\operatorname{median}\{\mathrm{g}(\mathrm{s}, \mathrm{t})\}
$$

$$
(s, t) \in S_{x y}
$$

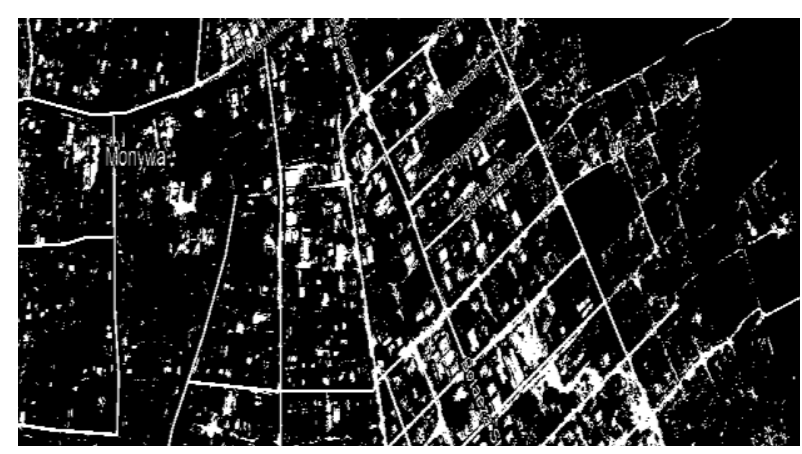

Fig.7. Median Filtering of Test Monywa Picture 


\section{III.(f)Morphological Operation}

Binary images (usually made up of pixels of two colors, black and white) have many disadvantages. Noise and structure can be destroyed, especially if binary spaces are built with simple properties. The purpose of image processing is to eliminate this disadvantage, taking into account the shape and structure of the image.[9]

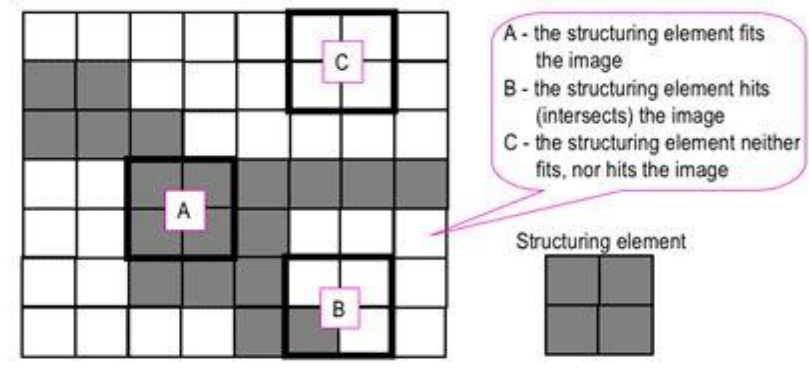

Fig. 8. Structural Element[10]

\section{III. (g)Morphological Erosion}

Erosion is used for shinking of element $\mathrm{X}$ by using element $\mathrm{Y}$ an dfor Sets $\mathrm{X}$ and $\mathrm{Y}$ in $\mathrm{Z} 2$, is defined by the following equation;[9]

$\mathrm{X} \ominus \mathrm{Y}=\left\{\mathrm{Z} /(\mathrm{Y})_{\mathrm{z}} \subseteq \mathrm{X}\right\}$

$\mathrm{X} \ominus \mathrm{Y}=\left\{\mathrm{Z} /(\mathrm{Y})_{\mathrm{z}} \sqcap \mathrm{X}^{\mathrm{c}} \neq \varnothing\right\}$

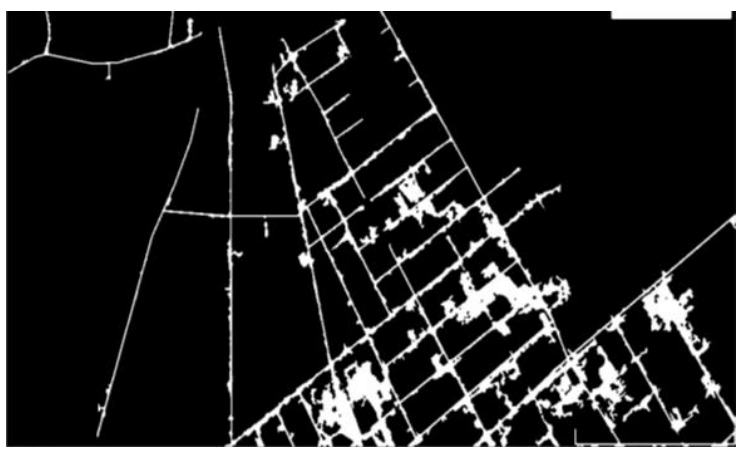

Fig. 9. Erosion of Test Monywa Picture

\section{III.(h)Morphological Dilation}

Erosion is the main operator of the shape sperm. This is usually used for photography. However, there are variants that work with gray images. The main effect of the operator on binary images is to remove the pixel boundaries (usually white pixels) in the foreground. Therefore, the reduced area of the foreground pixel is reduced. Reduces the holes in that part of the foreground pixel and increases the holes in those areas.[9]

Two data are used as input to corrupt the operator. The first image will be deleted the second part is a small coordinate element, usually called a structural element (Called Kernel).

This structural element is the input of an image that contributes to a certain erosion effect. The formula says; [9]
$\mathrm{X} \ominus \mathrm{Y}=\left\{\mathrm{Z} \in \mathrm{E} \backslash(\mathrm{Y})_{\mathrm{z}} \subseteq \mathrm{X}\right\}$

\section{EXPERIMENTAL RESULTS}

The results are based on five-year surveys as following table. In this section, the system user tests road change detection and uses old decade NASA Images described as Table 1.

TABLE I. Data Statement

\begin{tabular}{|l|l|l|}
\hline No. & \multicolumn{1}{|c|}{ Source } & Captured Time \\
\hline 1. & Google Earth Pro (Desktop App) & $1 / 1985$ \\
\hline 2. & Google Earth Pro (Desktop App) & $2 / 2002$ \\
\hline 3. & Google Earth Pro (Desktop App) & $12 / 2009$ \\
\hline 4. & Google Earth Pro (Desktop App) & $11 / 2015$ \\
\hline 5. & Google Earth Pro (Desktop App) & $7 / 2020$ \\
\hline Five datasets have 1116*632 spatial resolutions,
\end{tabular}
individually. Road change detection is shown in the following.

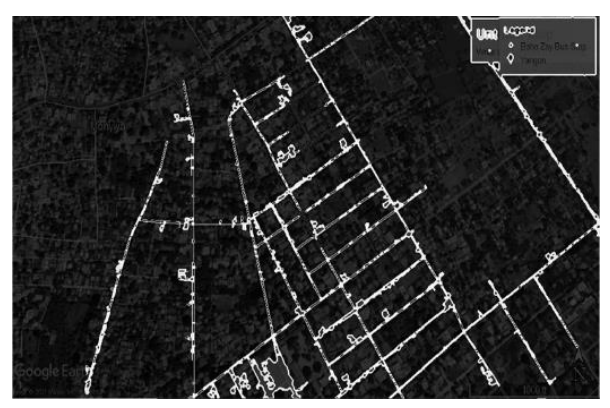

Fig.10. Road Change Detection at 1/1985

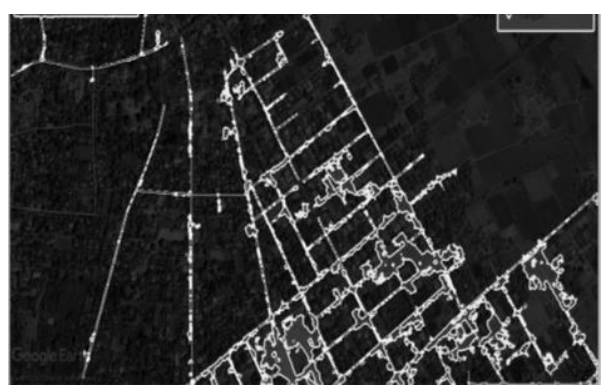

Fig.11. Road Change Detection at 2/2002

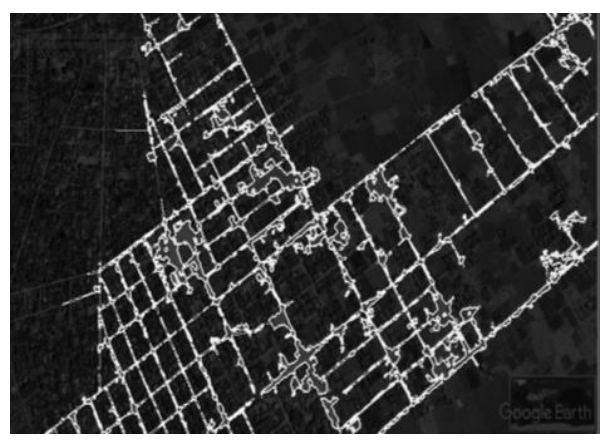

Fig.12. Road Change Detection at 12/2009 


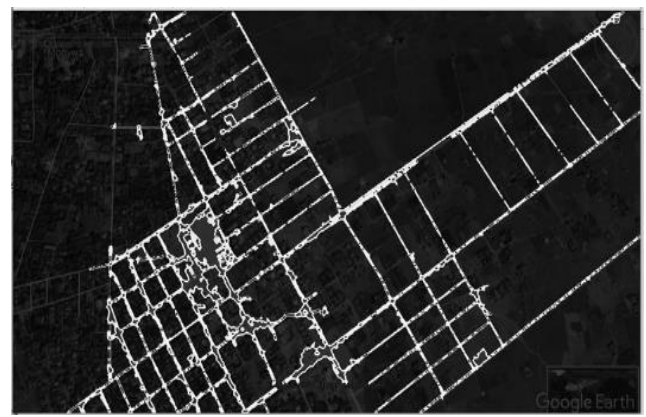

Fig.13. Road Change Detection at 11/2015

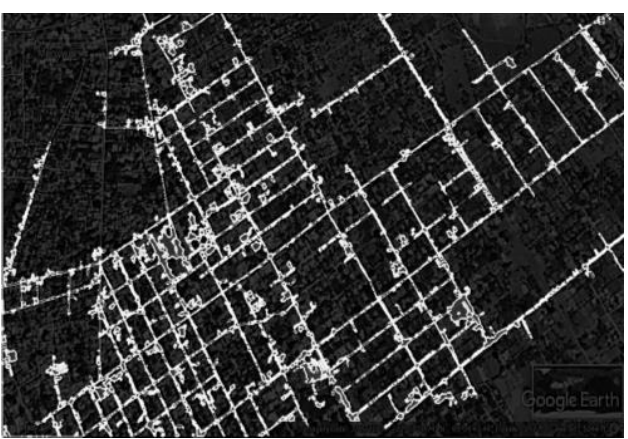

Fig.14. Road Change Detection at 7/2020

\section{CONCLUSION}

The point of this paper is to show you an effective system and method for finding paths from satellite imagery. The proposed algorithm for routing using morphological operations showed good results. By detecting at the improvement of roads, you can find out how much the city has improved. In this research, the user can find every highresolution image. Roads will continue to be explored based on real time.

\section{ACKNOWLEDGEMENTS}

The author of this journal would like to thank all the reviewers and thanks to the website sponsor.

\section{REFERENCES}

[1] M. Everingham, S. A. Eslami, L. Van Gool, C. K. Williams, J. Winn, and A. Zisserman," The pascal visual object classes challenge": A retrospective. International journal of computer vision, 111(1):98136, 2015.

[2] V. Iglovikov and A. Shvets. Ternausnet," U-net with vgg11 encoder pre-trained on imagenet for image segmentation." arXiv preprint arXiv:1801.05746, 2018.

[3] Yongyang Xu, Zhong Xie , Yaxing Feng and Zhanlong Chen," Road Extraction from High-Resolution Remote Sensing Imagery Using Deep Learning", Remote Sens. 2018, 10, 1461; doi:10.3390/rs10091461.

[4] Calimanut-Ionut Cira, and Ramón Alcarria, " A Deep LearningBased Solution for Large-Scale Extraction of the Secondary Road Network from High-Resolution Aerial Orth imagery", Appl. Sci. 2020, 10, 7272; doi:10.3390/app10207272.

[5] Korinne McDonnell/Glen Nagle," CANBERRA DEEP SPACE COMMUNICATION COMPLEX WEBSITE/ 2021".

[6] NASA official website, Kristen Erickson " NASA SPACE PLACE/March/ 2021"

[7] Sunil Bangare and Suhas Patil:"Reviewing Otsu's Method For Image Thresholding", published International Journal of Applied Engineering Research at May 2015.

[8] Average Median Filtering website 25 May 2010 is credited. https://www.cs.auckland.ac.nz/

[9] Ravi Srisha and Am Khan, " Morphological Operations for Image Processing: Understandings and its Applications", at December 2013.

[10] Mathematical morphology: from theory to applications, Laurent Najman and Hugues Talbot(Eds).ISTE-Wiley.ISBN 978-1-84821215-2.(520pp.)June 2010. 